Schmerz 2018 · 32:339-347

https://doi.org/10.1007/s00482-018-0284-8

Online publiziert: 21. März 2018

(c) Der/die Autor(en) 2018.

CrossMark
I. Gnass · S. Krutter · N. Nestler

Institut für Pflegewissenschaft und -praxis, Paracelsus Medizinische Privatuniversität, Salzburg, Österreich

\title{
Herausforderungen ambulanter Pflegedienste im Schmerzmanagement von Tumorpatienten
}

\section{Eine qualitative Untersuchung}

\section{Hintergrund und Fragestellung}

Schmerz ist ein häufig auftretendes Symptom bei Tumorpatienten [5]. Obwohl bei $90 \%$ der Tumorpatienten eine zufriedenstellende Schmerztherapie möglich wäre [17], berichten $50,7 \%$ unabhängig vom Krankheitsstadium und $66,4 \%$ in einem fortgeschrittenen oder terminalen Stadium über Schmerzen. Durch einen Wandel des Versorgungssystems und eine Erhöhung der Überlebensrate verlagert sich die Pflege und Behandlung von Tumorpatienten zusehends vom akutstationären in den häuslich ambulanten Bereich $[9,32]$. In der häuslichen Versorgung von Tumorpatienten kommt neben den Angehörigen und den Hausärzten den ambulanten Pflegediensten eine wichtige Rolle $\mathrm{zu}[2,27]$. Prävalenzraten zeigen, dass bei den 692.000 Pflegebedürftigen, die in Deutschland von 13.300 ambulanten Pflegediensten im häuslichen Setting versorgt werden [31], rund $70 \%$ unter Schmerzen leiden [20]. Eine zentrale Aufgabe der eingebundenen Pflegenden liegt dabei im multiprofessionellen Schmerzmanagement [32]. Pflegende agieren hier als Bindeglied zwischen Ärzten, Patienten und Angehörigen [34]. Durch ihre Nähe zum Patienten kommt Pflegenden auch in der Beobachtung des Schmerzerlebens der Patienten und der Wirksamkeit der Schmerzbehandlung eine tragende Rolle zu. Nach ärztlicher Anordnung führen sie schmerztherapeutische Maßnahmen aus, sie achten auf eine kontinuierliche
Anpassung der Schmerzversorgung und tragen zur Edukation der Patienten bei [9]. Inhaltlich beschrieben und festgelegt sind die Aufgaben Pflegender in der multiprofessionellen Schmerzversorgung in den Nationalen Expertenstandards "Schmerzmanagement in der Pflege“ $[10,12]$. Mit Inkrafttreten des PflegeWeiterentwicklungsgesetzes (SGB XI) im Jahr 2008 sind ambulante Pflegedienste zur Umsetzung der Nationalen Expertenstandards „Schmerzmanagement in der Pflege “ verpflichtet. In $\$ 112$ SGB XI wird wie folgt ausgewiesen:

Die zugelassenen Pflegeeinrichtungen sind verpflichtet, ... Expertenstandards nach $\$ 113$ a anzuwenden [7].

Lt. Bericht des Medizinischen Diensts des Spitzenverbands Bund der Krankenkassen (MDS) berücksichtigen 89,4\% der im Jahr 2013 geprüften Einrichtungen den Expertenstandard „Schmerzmanagement in der Pflege" bzw. stellten in Aussicht, konkrete Maßnahmen in dieser Hinsicht geplant zu haben [23].

Als Herausforderung in der Umsetzung des Schmerzmanagements in der ambulanten pflegerischen Versorgung bei Tumorpatienten werden in der Literatur Barrieren genannt, die mit einem fehlenden Wissen der Pflegenden zum Schmerzmanagement begründet werden [13, 28, 33]. Aber auch strukturelle Gründe, wie fehlende finanzielle Mittel [27] oder Schnittstellenproblematiken, z. B. aus dem Krankenhaus in die häusliche Pflege [32], sind anzuführen.
Für den deutschen Versorgungskontext ist mit der Trennung in die beiden Sozialgesetzbücher XI und V und den damit einhergehenden Barrieren in der Refinanzierung der Leistungen des Schmerzmanagements eine spezielle Herausforderung gegeben [6]. Zur Überwindung dieser Barrieren und für eine Verbesserung der multiprofessionellen Schmerzversorgung bei Tumorpatienten im häuslich ambulanten Bereich ist es wichtig, in der Evaluation des Schmerzmanagements neben der Sichtweise der Betroffenen auch die der professionellen Akteure einzubeziehen. In der Beschreibung der förderlichen und hinderlichen Aspekte in der ambulanten Schmerzversorgung von Tumorpatienten sollen Optimierungspotenziale für eine Verbesserung des multiprofessionellen Schmerzmanagements aufgezeigt werden. Vor diesem Hintergrund wird im Artikel folgender Frage nachgegangen:

Welche Herausforderungen erleben Pflegende ambulanter Pflegedienste in der Umsetzung eines systematischen Schmerzmanagements bei Menschen mit einer Tumorerkrankung?

\section{Methodik}

In der Arbeit werden Ergebnisse einer Teilstudie des Versorgungsforschungsprojekts „Aktionsbündnis Schmerzfreie Stadt Münster“ [26] präsentiert. 
Tab. 1 Beschreibung der Teilnehmer der fünf Gruppendiskussionen

\begin{tabular}{|c|c|c|c|}
\hline \multirow{2}{*}{$\begin{array}{l}\text { Thema der Gruppendiskussion It. } \\
\text { Ebene des Nationalen } \\
\text { Expertenstandards zum } \\
\text { „Schmerzmanagement in der } \\
\text { Pflege" }\end{array}$} & \multicolumn{2}{|c|}{ Geschlecht } & \multirow{2}{*}{$\begin{array}{l}\text { Anzahl der Teilnehmer je } \\
\text { Berufsgruppe bzw. } \\
\text { Tätigkeitsbereich }\end{array}$} \\
\hline & $\mathbf{M}$ & $\mathbf{W}$ & \\
\hline Schmerzeinschätzung & 2 & 5 & $\begin{array}{l}1 \times \text { Altenpflege } \\
2 \times \text { Pflegeleitung } \\
4 \times \text { Qualitätsmanagement }\end{array}$ \\
\hline Medikamentöse Schmerztherapie & 0 & 4 & $\begin{array}{l}2 \times \text { Gesundheits- und Krankenpflege } \\
1 \times \text { Altenpflege } \\
1 \times \text { Pflegeleitung }\end{array}$ \\
\hline $\begin{array}{l}\text { Nichtmedikamentöse Therapiemaß- } \\
\text { nahmen }\end{array}$ & 1 & 5 & $\begin{array}{l}2 \times \text { Gesundheits- und Krankenpflege } \\
2 \times \text { Pflegeleitung } \\
2 \times \text { Qualitätsmanagement }\end{array}$ \\
\hline $\begin{array}{l}\text { Schmerzmittelbedingte Nebenwirkun- } \\
\text { gen }\end{array}$ & 1 & 5 & $\begin{array}{l}2 \times \text { Gesundheits- und Krankenpflege } \\
2 \times \text { Pflegeleitung } \\
2 \times \text { Qualitätsmanagement }\end{array}$ \\
\hline Information, Schulung und Beratung & 1 & 3 & $\begin{array}{l}1 \times \text { Gesundheits- und Krankenpflege } \\
2 \times \text { Pflegeleitung } \\
1 \times \text { Qualitätsmanagement }\end{array}$ \\
\hline
\end{tabular}

\section{Datenerhebung}

Der explorative Charakter der Forschung und das qualitative methodische Vorgehen sollen dazu beitragen, Herausforderungen in der Umsetzung des gesetzlich vorgeschriebenen Nationalen Expertenstandards „Schmerzmanagement in der Pflege" aus Sicht der ambulant Pflegenden zu beschreiben. Die Erhebung fand in fünf leitfadengestützten Gruppendiskussionen [4] statt, die thematisch auf die fünf Ebenen des zum Erhebungszeitraum gültigen Expertenstandards „Schmerzmanagement in der Pflege bei akuten oder tumorbedingten chronischen Schmerzen" [11] Schmerzeinschätzung, medikamentöse und nichtmedikamentöse Schmerztherapie, schmerzmittelbedingte Nebenwirkungen sowie Information, Schulung und Beratung fokussierten. Die Inhalte der Interviewleitfäden wurden der jeweils $\mathrm{zu}$ diskutierenden Ebene thematisch angepasst. Im Sinne eines qualitativen induktiven Vorgehens hatten die Teilnehmer der Gruppendiskussionen auch die Möglichkeit, weitere Themen in die Diskussion einzubringen.

Die Auswahl der teilnehmenden ambulanten Pflegedienste erfolgte als Gelegenheitsstichprobe. Von den zum Erhebungszeitraum in Münster insgesamt vorhandenen 36 Pflegediensten stimmten 14 einer Teilnahme am Projekt sowie an den Gruppendiskussionen zu. Die Einrichtungen, wovon 6 in öffentlichrechtlicher und 8 in privater Trägerschaft geführt wurden, glichen sich in ihrer Versorgungsstruktur sowie ihrem -angebot. Die Auswahl der Teilnehmer für die Gruppendiskussionen wurde in allen 14 ambulanten Pflegediensten von der Kontaktperson in der jeweiligen Einrichtung vorgenommen. Dadurch ergab sich eine heterogene Zusammensetzung der Gruppen: Neben Pflegenden mit einer dreijährigen Gesundheits- und Krankenpflegeausbildung oder Altenpflegeausbildung nahmen auch leitende Mitarbeiter sowie Qualitätsmanagementbeauftragte an den Diskussionen teil (• Tab. 1). Die Gruppengröße betrug durchschnittlich fünf Teilnehmer, wobei manche Personen an mehreren Gruppendiskussionen teilnahmen.

Die Gruppendiskussionen (Dauer 1-1,5 h) fanden von Juli bis September $2012 \mathrm{im}$ Gesundheitshaus in Münster statt. Die Interviews wurden von einer erfahrenen Pflegewissenschaftlerin in einem nondirektiven Moderationsstil durchgeführt, um einen selbstläufigen Diskurs zu befördern. Den Teilnehmern der Gruppendiskussionen war es dadurch möglich, weitere Themen in die Diskussionen einzubringen bzw. die in den Interviewleitfäden thematisierten Inhalte $\mathrm{zu}$ vertiefen. Eine weitere Projektmitarbeiterin führte parallel ein
Ablaufprotokoll. Alle Gruppendiskussionen wurden auf Tonband aufgezeichnet und im Anschluss transkribiert.

\section{Datenauswertung}

Die Analyse der Gruppendiskussionen folgte der qualitativen Inhaltsanalyse nach Mayring [22] mit dem Ziel, die manifesten Aussagen der Diskussionsteilnehmer thematisch zu systematisieren und inhaltlich zusammenzufassen. Für die Auswertung der Daten wurden vorab Kategorien deduktiv festgelegt, die sich an den fünf Ebenen des Nationalen Expertenstandards „Schmerzmanagement in der Pflege“ [11] orientierten. Im Laufe der Analyse wurden weitere Subkategorien gebildet, die sich in induktiver Weise aus den Inhalten der Interviews ergaben. Die Auswertung erfolgte unabhängig durch zwei Projektmitarbeiterinnen mit Erfahrung in der qualitativen Datenanalyse unter Zuhilfenahme der Analysesoftware MAXQDA 10.

Im Sinne der methodischen Güte qualitativer Forschung [21] wurde der Prozess der Datenerhebung und -analyse dokumentiert sowie in der Forschungsgruppe regelmäßig reflektiert, kritisch überprüft und diskutiert. Die Methode der qualitativen Inhaltsanalyse ermöglichte eine regelgeleitete Auswertung der Daten. Die Interpretation der Daten erfolgte gemeinsam im Forschungsteam, bestehend aus erfahrenen Pflegewissenschaftlern mit Expertise im pflegerischen Schmerzmanagement.

\section{Ethik}

Die Westfälische Wilhelms-Universität Münster stellte dem Projekt ein positives Ethikvotum aus (2010-010-f-S). Alle Interviewten nahmen freiwillig teil und erklärten zu Beginn der Gruppendiskussion schriftlich ihre informierte Einwilligung.

\section{Ergebnisse}

Die Ergebnisse werden gebündelt entlang der fünf Gruppeninterviews und der darin behandelten Haupt- bzw. Subkategorien dargestellt (• Abb. 1). 


\section{Schmerzeinschätzung}

Das Wissen zur Schmerzeinschätzung und die Unterscheidung in initiale und differenzierte Schmerzeinschätzung werden von den teilnehmenden Pflegenden für das Erstgespräch hervorgehoben und es wird die Erfahrung berichtet, dass die Interpretation der Schmerzintensität bei Patienten mit immer wieder gleich angegebener Schmerzstärke schwierig ist. Obwohl die Mehrdimensionalität des Schmerzes bei Patienten mit Tumorerkrankung erlebt wird, ist eine systematische Erfassung der Dimensionen, z.B. der psychosozialen Aspekte, nicht etabliert.

Es ist so ein Erleben des ganzen Schmerzes, so ein Universalschmerz ... körperliche Schmerzen und existenzielle Ängste ... Wir sollten uns davon verabschieden, all das, was Menschen bewegen mag, wenn sie erkennen und realisieren, dass sie sich in ihrer letzten Lebensphase befinden, an einer Zahl festzumachen. Das auf eine Nummer zu bringen ist im Sinne der Rangskala so wohl gar nicht möglich (GD1, 97-99).

Im Zusammenhang mit der Schmerzeinschätzung werden zielgruppenspezifische Einschätzungs- und Dokumentationsmaterialien wie die numerische Rating-Skala (NRS) als Standard benannt und Protokollblätter oder Schmerztagebücher angeführt. Die Forderung vom Medizinischen Dienst der Krankenversicherung (MDK), Beobachtungsskalen bei Menschen mit kognitiven Beeinträchtigungen anzuwenden, wird als gerecht gewertet.

Pflegende beschreiben die Notwendigkeit für ein umfängliches Wissen zur Schmerzeinschätzung, zugleich berichten sie von einer Abwehr der Durchführung, da sie mit dem Ergebnis der Schmerzeinschätzung nicht immer eine handlungsorientierte oder therapeutische Konsequenz seitens der Hausärzte erfahren.

Die Zusammenarbeit mit den Hausärz- ten ist nicht so besonders gut. Und da sind uns auch die Hände gebunden. Sie [Anm.: die Hausärzte] schauen auf keine Skala und sie schauen auch nicht auf das, was wir dokumentieren, das interessiert sie meistens nicht. Wir müssen ständig hinterher telefonieren. Dass die Ärzte das dann häufig gar nicht ernst nehmen und sagen: „Ja, Frau Sowieso, die kenne ich schon mein Leben lang, sie jammert immer ein bisschen", wird das häufig abgetan (GD2, 360-367).

Pflegende in ambulanten Diensten sehen die Schwierigkeit, den Zusammenhang zwischen der Umsetzung des gesamten Schmerzmanagements und dem jeweilig vereinbarten pflegerischen Versorgungsauftrag herzustellen. Dies wird deutlich in der Schmerzverlaufserfassung, die vor dem Hintergrund der Anwesenheitszeiten von Pflegenden beim Patienten als schwierig bis gar nicht umsetzbar erlebt wird. Die Herausforderung besteht darin, eine notwendige Schmerzerfassung über den Tagesverlauf sicherzustellen, wofür

Hier steht eine Anzeige. 
Schmerz 2018 · 32:339-347 https://doi.org/10.1007/s00482-018-0284-8

(c) Der/die Autor(en) 2018.

\section{Gnass · S. Krutter · N. Nestler}

\section{Herausforderungen ambulanter Pflegedienste im Schmerzmanagement von Tumorpatienten. Eine qualitative Untersuchung}

\section{Zusammenfassung}

Hintergrund. Menschen mit Tumorerkrankungen werden zunehmend ambulant versorgt. Schmerz ist ein relevantes Begleitsymptom, an dessen Behandlung auch Pflegende ambulanter Pflegedienste beteiligt sind. In den Nationalen Expertenstandards "Schmerzmanagement in der Pflege" werden evidenzbasierte Empfehlungen zur Umsetzung eines adäquaten Schmerzmanagements ausgesprochen. Vor den gegenwärtigen strukturellen Gegebenheiten der ambulant pflegerischen Versorgung beschreiben Pflegende sowohl Barrieren als auch Herausforderungen in der Umsetzung. Methoden. Mittels 5 leitfadengestützter Gruppendiskussionen wurden Pflegende 14 ambulanter Pflegedienste zu den erlebten
Herausforderungen im Schmerzmanagement befragt. Die Befragung fokussiert auf die Umsetzung jeweils einer Empfehlungsebene: Schmerzeinschätzung, medikamentöse Schmerztherapie, nichtmedikamentöse Maßnahmen, schmerzmittelbedingte Nebenwirkungen sowie Information, Schulung und Beratung in der Versorgung von Menschen mit Tumorerkrankung. Die Daten wurden inhaltsanalytisch ausgewertet.

Ergebnisse. Einerseits besteht ein Bedarf an weiteren Kenntnissen und Möglichkeiten, z. B. zur Erfassung des Schmerzes als multidimensionales Phänomen. Andererseits sind Bedingungen für eine kontinuierliche schmerzbezogene Begleitung von Tumorpatienten in der ambulant pflegerischen
Versorgung nur bedingt gegeben. Eine notwendige zeitnahe Abstimmung im Behandlungsteam stellt sich mit den Hausärzten schwieriger dar als in der Zusammenarbeit mit dem Palliativnetz. Die Einbindung von Angehörigen hat für die Sicherstellung der Versorgung einen hohen Stellenwert. Schlussfolgerungen. Neben Kenntnissen und Kompetenzen zu den pflegerischen Aufgaben sind Strukturen und Prozesse im interprofessionellen Schmerzmanagement weiter aufzubauen.

\section{Schlüsselwörter}

Tumorschmerz - Ambulante Pflege .

Schmerzmanagement · Palliativversorgung . Qualitative Forschung

\section{Challenges for home care services in the pain management of cancer patients. A qualitative study}

\section{Abstract}

Background. People with cancer are increasingly supported by home care services. Pain is a relevant symptom of these diseases and nurses of home care services are involved in the treatment. The German National Expert Standard "Pain management in nursing" includes evidence-based recommendations for the implementation of adequate pain management. Considering the given structural conditions of home care services, nurses describe both barriers and challenges with the implementation.

Methods. By means of five guidelinebased discussion groups, nurses of 14 home care services were questioned about the challenges they had experienced in pain management. The questioning focuses on the level of implementation of the recommendation for each aspect: pain assessment, pharmacological pain therapy, non-pharmacological pain therapy, painrelated side effects, information, training, and counseling in the care of people with cancer. A qualitative content analysis was conducted. Results. On the one hand, the results illustrate a need for further knowledge and possibilities, e.g., for the assessment of pain as a multidimensional phenomenon and, on the other hand, that the conditions for continuous pain monitoring of cancer patients in home care services are limited. The need for short-term reconciliation with the treatment team and the practitioners proved to be more difficult than the cooperation with the palliative care network. Involvement of family members is important to ensure uninterrupted treatment.

Conclusions. Beside knowledge and competencies regarding nursing care, structures and processes for interprofessional pain management need further development and research.

Keywords

Cancer pain - Home care service - Pain management · Palliative care · Qualitative research zum Teil Angehörige oder ein zusätzlicher Kollege mit eingebunden werden müssen.

\section{Medikamentöse Schmerztherapie}

Das Wissen zur medikamentösen Schmerztherapie wird als höchst relevant für den Umgang mit chronisch kranken Schmerzpatienten berichtet und der Wunsch nach „besser geschulten“ Hausärzten geäußert. Pflegende erleben, dass Hausärzte die Schmerzproblematik und
Informationen kaum ernst nehmen und sich die Kommunikation durch unterschiedliche Arbeitszeitbedingungen zwischen den Pflegenden und Ärzten schwierig gestaltet. Geforderte interprofessionelle Verfahrensregelungen zur medikamentösen Schmerztherapie sind durch hohe notwendige Abstimmungsprozesse gekennzeichnet und selten bis gar nicht schriftlich fixiert.

Pflegende nehmen die Rolle des vehementen „Vermittlers“ (GD2, 110) für die Belange der Patienten ein und beschrei- ben eine z.T. unangemessene und unkoordinierte Versorgung in der Zusammenarbeit mit Hausärzten, sodass der Prozess zur Abstimmung der medikamentösen Schmerztherapie an jeder Stelle scheitern kann. Die kontinuierliche Sicherstellung und Anpassung der medikamentösen Schmerztherapie ist durch den Versorgungsauftrag sowie dessen zeitliche Taktung erschwert und kann z. T. nur durch Einbeziehung der Angehörigen gewährleistet werden, Stichwort: präventive Schmerzmittelgabe. 
Bei der Einbindung eines ortsansässigen Palliativnetzes (SAPV) erfahren Pflegende hingegen Koordination und einen Handlungsspielraum in der Therapieanpassung, z. B. bei Schmerzspitzen. Medikamente sind als Basis oder Bedarf angeordnet und auch für weitere Symptome, z. B. Übelkeit oder Unruhe, entsprechend vorrätig.

Mit dem Palliativnetz ist es wirklich immer abgestimmt. Dadurch dass dieser ständige Kontakt zum Palliativnetz da ist, handelt man niemals irgendwo eigenmächtig, sondern das ist immer in Abstimmung ... Die Pfleger vom Palliativnetz stimmen eine Änderung der Medikation mit den Ärzten ab ... Das wird auch so durchgeführt und es geht auch schneller... Ja genau, das ist der Punkt, es geht schneller (GD2, 317-322).

\section{Schmerzmittelbedingte Nebenwirkungen}

Qualitätsmanagementbeauftragte erleben eine geringe Sensibilisierung und gleichzeitig eine große Herausforderung für die Vermittlung von Wissen $z u$ schmermittelbedingten Nebenwirkungen bei den Mitarbeitern. Pflegende ihrerseits beschreiben die Schwierigkeit in der Unterscheidung zwischen schmerzmittelbdingten Nebenwirkungen und Symptomen der Tumorerkrankung.

Schwieriger handzuhaben sind teilweise diese etwas unspezifischen Symptome, die möglich sind, die von vielerlei herkommen können. Von anderen Medikamenten, vielleicht von der Erkrankung an sich. $\mathrm{Da}$ ist dann eine Schwierigkeit da, die [Anm.: die Nebenwirkungen] richtig zu erfassen, und die Schwierigkeit, alle auf den Stand des Wissens zu kriegen (GD4, 1049-1058).

Die Erfassung und Dokumentation findet nicht systematisch, sondern zumeist erst mit dem Auftreten von Symptomen statt und die Erforschung der Ursachen kann zwei bis drei Tage dauern. Bei „unklaren“ Symptomen verweisen Pflegende immer auf die Kommunikation mit dem Hausarzt und nehmen ggf. selber auch Abspra- chen mit dem Hausarzt vor. Hier wird erneut auf die Problematik der zeitnahen Rückmeldung hingewiesen.

\section{Nichtmedikamentöse Therapie- maßnahmen (NMT)}

In den Pflegediensten wird kein einheitliches und umfängliches Wissen zu nichtmedikamentösen Maßnahmen beschrieben. Pflegende erleben sich nicht als Experten für die Anwendung von NMT, erwarten von sich selbst aber gleichzeitig einen professionellen Umgang für deren Einsatz. Die Fähigkeiten einzelner Pflegender und Fortbildungen fördern die Umsetzung von spezifischen NMT, z.B. Aromapflege, und die Sensibilisierung aller Mitarbeitenden. Die Benennung der vielseitigen Angebote an Patienten und Bezugspersonen, wie in einem „Bauchladen“ (GD4, 150) beschrieben, beinhalten zugleich jene Angebote, die vom Hausarzt angeordnet werden müssen, z. B. schmerzlindernde Salben. Pflegende erleben sich in einer Zwickmühle, einerseits hochqualifiziert NMT an- 
zuwenden, diese aber nicht umsetzen zu können, sodass sie sich letztendlich lediglich in einer beratenden Rolle sehen. Für die Sicherstellung der Anwendung von NMT ist auch hier eine starke Einbindung von Angehörigen notwendig.

Also Massagen zum Beispiel ... Ich bin auch Massagetherapeutin. Und ich finde das ist nicht zu unterschätzen, dass man da auch ganz schnell etwas falsch machen kann ... wenn man das nicht wirklich professionell gelernt hat. Ich denke, dass eine Krankenpflegekraft das nicht einfach so ohne Weiteres machen sollte... Und das andere ist der Zeitaufwand ... das muss ja dann auch abgedeckt sein (GD3, 531-552).

\section{Information, Schulung und Beratung}

Der Aufforderung zum Vorhalten von Unterlagen zu Information, Schulung und Beratung zum Schmerz und Schmerzmanagement wird von den ambulanten Pflegediensten vereinzelt mit einer Broschüre begegnet. Zumeist „verschwindet“ diese Broschüre bei den Patienten „im Zeitungsstapel“ (GD5, 1195) und findet keine weitere Beachtung. Notwendige Schulungskompetenzen der Pflegenden für eine zielgruppenspezifische Vorgehensweise zum Schmerzmanagement sind eine große Herausforderung, derer sich ambulante Pflegedienste annehmen wollen. Pflegende binden im Rahmen ihrer Möglichkeiten und trotz zeitlicher Restriktionen Angehörige in die Bemühungen ein.

Eine Gewährleistung für Information, Schulung und Beratung für Patienten kann aufgrund fehlender zielgruppenspezifischer Konzepte nicht erfüllt werden. Pflegende beschreiben, dass sich individuelle Bedarfe im Gespräch in der jeweiligen Pflegesituation ergeben und sie die Bereitschaft der Patienten eher als nicht interessiert bis ablehnend erleben. Für Pflegende besteht eine Schwierigkeit darin, ablehnendes Verhalten bzw. fehlendes Verständnis von Patienten, auch mit vorhandenen Schmerzen, in der Patientendokumentation festzuhalten.

\section{Diskussion}

Die vorliegende qualitative Untersuchung ist der Frage nachgegangen, welche Herausforderungen Pflegende ambulanter Pflegedienste in der Umsetzung eines systematischen Schmerzmanagements bei Menschen mit einer Tumorerkrankung im häuslich ambulanten Setting erleben.

Dabei wurde das Ziel verfolgt, die fördernden und hemmenden Faktoren in der Umsetzung des gesetzlich vorgeschriebenen Nationalen Expertenstandards „Schmerzmanagement in der Pfle-

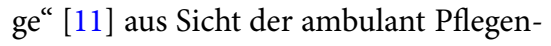
den zu beschreiben.

In Bezug auf die Ebene der Schmerzerfassung wird in den Gruppendiskussionen deutlich, dass die Pflegenden den Anspruch haben, die Schmerzsituation von Tumorpatienten individuell zu erheben und den Schmerzverlauf kontinuierlich zu dokumentieren. Wahrgenommen, aber nicht mit entsprechenden Instrumenten erhoben werden dabei die multidimensionalen Aspekte im Schmerzerleben, die besonders dem Tumorschmerz $\mathrm{zu}$ eigen sind [24]. Die von den Pflegenden genutzte NRS wird für die Einschätzung von Tumorschmerz als unzureichend eingeschätzt. Die Pflegenden berichten von Schwierigkeiten, die bei Patienten im Umgang mit der NRS zu beobachten sind, insbesondere in der wiederholten Einschätzung von Schmerzen. Ein Umstand, der auch in anderen Studien und für andere Versorgungssettings beschrieben wird [14, 15, 19]. Die Pflegenden in dieser Stichprobe äußern ferner eine Zurückhaltung in der standardisierten Messung der Schmerzen, die sie mit dem Ausbleiben einer Konsequenz vonseiten der Hausärzte begründen.

In anderen Untersuchungen im ambulanten Setting wird ein Nicht-nachSchmerzen-Fragen oder Nicht-aufSchmerzäußerungen-von-Patienten-Reagieren als passive Haltung empirisch und theoretisch erörtert [3,9]. Die darin zusätzlich gegebene Begründung, dass auch ein Mangel an Wissen, ein Fehlen von Kompetenz und eine unklare Rollenbeschreibung der ambulant Pflegenden zu einer Zurückhaltung in der Schmerzerfassung führen können, kann auch für die von uns befragten Pflegenden als weitere mögliche Erklärung herangezogen werden.

Herausforderungen im Schmerzmanagement, die sich aus der interdisziplinären Zusammenarbeit mit Hausärzten ergeben, werden von den ambulant Pflegenden aber auch für die Ebene der medikamentösen Schmerztherapie zum Ausdruck gebracht. Auch hier führt das Erleben der Pflegenden, dass auf die von ihnen mitgeteilten Informationen zur Schmerzsituation der Patienten von den Hausärzten oftmals nicht zeitnah und auch nicht immer angemessen reagiert wird, zu einer Passivität im interprofessionellen Austausch. Ein Ergebnis, das sich auch in anderen Studien wiederfindet, in denen die Schnittstelle der ambulant Pflegenden mit den Hausärzten ebenso als problematisch beschrieben wird. Die in den Studien dargestellte ablehnende Haltung der Hausärzte in der Schmerzversorgung, die fehlende Bereitschaft Schmerzmittel zu verschreiben $[2,33]$, ein eingeschränktes Wissen und die Schwierigkeit der Kontaktaufnahme mit Hausärzten [13] decken sich gleichsam mit den Ergebnissen unserer Gruppendiskussionen mit ambulant Pflegenden. Erschwert wird dadurch die Rollenausübung der ambulant Pflegenden als Vermittler im Schmerzmanagement zwischen Patienten, Angehörigen und Hausärzten [9, 34], in der sich auch die von uns Befragten sehen.

Weshalb die Rolle als Vermittler mit hohem Aufwand und erheblichen Hindernissen verbunden ist, kann auf den interprofessionellen Ansatz des pflegerischen Schmerzmanagements zurückgeführt werden [10], in dem die Pflegenden auf die Abstimmung und Kooperation mit den Hausärzten angewiesen sind. Gestaltet sich die Zusammenarbeit mit Hausärzten als herausfordernd, so gestaltet sich folglich auch das pflegerische Schmerzmanagement als herausfordernd. Ein Misslingen der Zusammenarbeit mit Hausärzten kann bei ambulant Pflegenden dazu führen, zurückhaltend zu agieren im Erkennen von und Reagieren auf Schmerzen der Patienten, so wie das auch im theoretischen Modell von Blomberg und Kollegen beschrieben wird [3]. Von den von uns befragten ambulant 
Pflegenden wird hingegen die Zusammenarbeit im Schmerzmanagement mit dem Palliativnetz in der spezialisierten ambulanten Palliativversorgung (SAPV) positiv eingeschätzt, so wie das auch Ergebnis anderer Untersuchungen ist [2]. Hier können ambulant Pflegende aktiv agieren und sind mit ihrem Beitrag zur Schmerzversorgung zufrieden.

Herausforderungen im Schmerzmanagement, die ambulant Pflegende auf der Ebene der schmerzmittelbedingten Nebenwirkungen erleben, z.B. die Schwierigkeit der Unterscheidung der Ursache einer Nebenwirkung [30], nehmen, so wie die Herausforderungen in der medikamentösen Schmerztherapie, ihren Ausgang oftmals in der interdisziplinären Zusammenarbeit mit Hausärzten. Denn auch hier sind die ambulant Pflegenden auf den Austausch und die Kooperation mit den Hausärzten angewiesen, die, wie zuvor bereits diskutiert, als verbesserungswürdig einzustufen sind.

Die Planung und Durchführung von NMT erscheinen hingegen weitestgehend monodisziplinär rein pflegerisch bedingt. Gleichwohl werden dabei ein Mangel an Wissen zum gezielten Einsatz der NMT sowie das Fehlen einer systematischen und einheitlichen Anwendung im Team angeführt. Ferner fehlt es den Pflegenden an Evaluationsmaßnahmen zur regelgeleiteten Bewertung und Anpassung der angewandten NMT, so wie es der Pflegeprozess vorsieht.

Die letzte thematische Ebene des Schmerzmanagements betrifft Information, Schulung und Beratung. Hierzu wird in den Gruppendiskussionen ein deutlicher Mangel in der pflegerischen Unterstützung der Tumorpatienten und ihrer Angehörigen beschrieben. Kritisch angemerkt wird eine teilweise fehlende Kompetenz für das Angebot edukativer Maßnahmen, fehlende zeitliche Ressourcen und auch die zurückhaltende Annahme vonseiten der Patienten und ihrer Angehörigen. Es bedarf einer Verbesserung der Edukation zur Schmerzversorgung im ambulanten Bereich, was bereits andernorts diskutiert wird [8, 28]. Der gezielte Einsatz von Beratungen bei Tumorpatienten im häuslichen Bereich bedingt Erfolge in der Einstellung der
Patienten zum Schmerzmanagement [1]. In der Literatur vorgeschlagen werden bereits früh im Verlauf der Krankheit anzusetzende Schulungsmaßnahmen bei Tumorpatienten, die nicht nur dazu dienen, das Wissen zum Schmerzmanagement $\mathrm{zu}$ erhöhen, sondern auch die selbstständige Anwendung therapeutischer Maßnahmen zu steigern und den kommunikativen Austausch mit den Professionellen zu fördern [25]. Als weitere Themen von Schulungen und Beratung werden die Fähigkeit zur Selbstbeobachtung des Schmerzes, die Anwendung von NMT sowie die Bewältigung von Schmerzen in der Literatur vorgeschlagen [9].

Den ambulant Pflegenden kommt im Schmerzmanagement von Tumorpatienten in der ambulanten Versorgung eine zentrale Rolle in der Koordination und

\section{Hier steht eine Anzeige.}

\section{贶 Springer}

Umsetzung des Schmerzmanagements zu. Ein gutes Gelingen des interprofessionellen Schmerzmanagements im häuslich ambulanten Setting ist dabei abhängig von der optimalen kommunikativen Abstimmung zwischen den ambulant Pflegenden und den Hausärzten [16], die auch in anderen Versorgungszusammenhängen als , chronischer Konflikt" dargestellt wird [18]. Für die Überwindung der Herausforderungen im Schmerzmanagement von Tumorpatienten sind jedoch auch die ambulant Pflegenden selbst angehalten, ihr Wissen zur Schmerzversorgung zu erweitern, in der Schmerzerfassung multidimensionale Instrumente heranzuziehen sowie die Anwendung von NMT gezielt, systematisch und einheitlich umzusetzen.

Zur Sicherstellung des interprofessionellen Schmerzmanagements, in das 
auch ambulante Pflegedienste involviert sind, muss es Anpassungen in den Leistungskatalogen für die ambulanten Pflegedienste im Rahmen des SBG XI geben. Ferner wäre für die Umsetzung des Schmerzmanagements eine angemessene gesetzliche Rahmenbedingung auch im SBG V zu berücksichtigen. So könnte ein neu definiertes Abrechnungssystem von interprofessionellen Leistungen im Schmerzmanagement für ambulante Pflegedienste eine gesicherte Refinanzierung ermöglichen. In diesem Zusammenhang wäre dann auch die Ausbildung ambulant Pflegender zu pflegerischen Schmerzexperten $\mathrm{zu}$ forcieren, damit diese ihrer so wichtigen Rolle als Vermittler im Schmerzmanagement gerecht werden und diese aktiv ausüben können. Die Rolle des pflegerischen Schmerzexperten ist fester Bestandteil des Expertenstandards „Schmerzmanagement in der Pflege bei chronischen Schmerzen" [12]. Pflegeteams in ambulanten Pflegediensten, Einrichtungen der stationären Altenhilfe und auch im Krankenhaus werden angehalten, bei komplexeren Versorgungszusammenhängen im Schmerzmanagement spezifisch ausgebildete „pflegerische Schmerzexperten“ einzubeziehen, um den Herausforderungen von mono- wie auch interprofessionellen/-disziplinären Behandlungsansätzen gerecht zu werden. Das Konzept der SAPV, das eine kontinuierliche Versorgung über $24 \mathrm{~h}$ und 7 Tage die Woche vorsieht und für das Pflegende in ambulanten Pflegediensten eine fachlich kompetente Zusammenarbeit beschreiben, könnte als wünschenswerter Ansatz für eine systemische Einmündung in die Versorgungsstruktur der ambulanten Pflegedienste dienen.

Um weitere Erkenntnisse für die interprofessionelle Zusammenarbeit für die Schmerzversorgung von Menschen mit Tumorerkrankungen in der ambulanten Versorgung zu erhalten, besteht ein Forschungsbedarf, der die Perspektive der Haus-/Fachärzte und ggf. Hospiz- und/ oder SAPV-Teams untersucht. Untersuchungen von Modellvorhaben ( $\$ 63$ Absatz 3c SGB V) zum Einsatz von pflegerischen Schmerzexperten im Versorgungsgefüge könnten zudem weitere
Erkenntnisse für notwendige Anpassungen der Rahmenbedingungen aufzeigen [29]. Im Rahmen von SGB-XI-Leistungsverträgen sind regelhaft auch Pflegehilfskräfte (einjährig examiniert) in der ambulant-pflegerischen Versorgung tätig. Es muss daher kritisch hinterfragt werden, welche Aspekte des pflegerischen Schmerzmanagements, das sich laut Expertenstandard an Pflegefachkräfte (dreijährig examiniert) richtet, in die Durchführungsverantwortung von Pflegehilfskräften gegeben werden können.

\section{Limitationen}

Limitationen der Studie ergeben sich ggf. aus dem geringen Umfang der Stichprobe und der Begrenzung der Studie mit ambulanten Pflegediensten auf eine Stadt. Die Ergebnisse können daher auch nicht als repräsentativ betrachtet werden. Inhaltliche Verdichtungen sind in den Ergebnissen zwar gegeben, eine theoretische Sättigung wurde jedoch vermutlich nicht erreicht. Die Ausweitung auf ambulante Dienste in anderen Regionen sowie eine noch gezieltere Zusammensetzung der Teilnehmer der Gruppendiskussionen könnten die Ergebnisse daher ergänzen. Auch die Verwendung einer anderen Analysemethode (z. B. dokumentarische Methode) könnte die Ergebnisse zusätzlich vertiefen. Wie für qualitative Studien üblich kann nicht ausgeschlossen werden, dass der Verlauf der Diskussionen und die Gruppenzusammensetzungen einen Einfluss auf die erhobenen Daten hatten. Es erfolgte eine Präsentation der Ergebnisse in den teilnehmenden ambulanten Diensten, die auf $\mathrm{Zu}$ stimmung stieß und keine Fehlschlüsse aufzeigte. Da eine intensive Diskussion der Ergebnisse mit den Befragten jedoch unterblieb, kann dabei nicht von einer kommunikativen Validierung gesprochen werden.

\section{Fazit für die Praxis}

\footnotetext{
- Mitarbeiter von ambulanten Pflegediensten sollten relevante Parameter zur Schmerzsituation während der Versorgung erfassen, dokumentieren und kommunizieren.
}

- Die Kontinuität der Erfassung von relevanten Parametern zur Schmerzsituation ist abhängig vom vereinbarten Leistungsumfang/-vertrag und bedarf einer kritischen Betrachtung.

- Kenntnisse und Kompetenzen pflegerischer Aspekte im Schmerzmanagement sind weiter aufzubauen, z. B. durch interprofessionelle Ausund Fortbildungsangebote.

- Zur Sicherung der Kontinuität der Schmerzversorgung bedarf es regelhafter und festgeschriebener interprofessioneller Abstimmungen mit Haus-/Facharzt und ggf. Hospizund/oder SAPV-Team.

- Für die Etablierung von relevanten Handlungen von Pflegenden ambulanter Pflegedienste, die zur Optimierung der Schmerzsituation bei Mensch mit Tumorerkrankung im Rahmen des interprofessionellen Schmerzmanagements beitragen, ist eine Anpassung von Strukturen und gesetzlichen Rahmenbedingungen erforderlich.

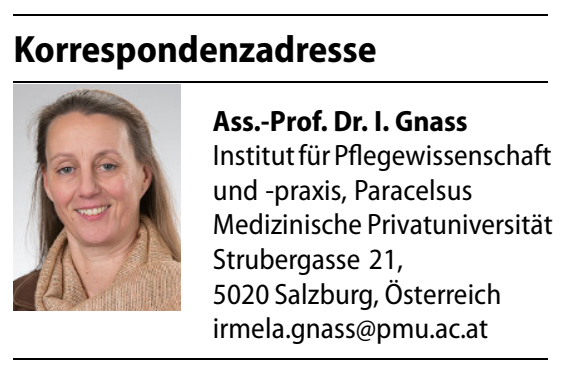

Funding. Open access funding provided by Paracelsus Medical University.

\section{Einhaltung ethischer Richtlinien}

Interessenkonflikt. I. Gnass, S. Krutter und N. Nestler geben an, dass kein Interessenkonflikt besteht.

Dieser Betrag beinhaltet keine von den Autoren durchgeführte Interventionsstudie an Menschen oder Tieren.

Open Access Dieser Artikel wird unter der Creative Commons Namensnennung 4.0 International Lizenz (http://creativecommons.org/licenses/by/4.0/deed. de) veröffentlicht, welche die Nutzung, Vervielfältigung, Bearbeitung, Verbreitung und Wiedergabe in jeglichem Medium und Format erlaubt, sofern Sie den/die ursprünglichen Autor(en) und die Quelle ordnungsgemäßnennen, einen Linkzur Creative Commons Lizenz beifügen und angeben, ob Änderungen vorgenommen wurden. 


\section{Literatur}

1. Aubin M, Vézina L, Parent R et al (2006) Impact of an educational program on pain management in patients with cancer living at home. Oncol Nurs Forum 33:1183-1188

2. Berendt J, Papke J, Mitzscherlich B et al (2015) Spezialisierte ambulante Palliativversorgung aus Sicht ambulanter Pflegedienste - Eine empirische Untersuchung in Sachsen-Anhalt Ost. ZPalliativmed 16:117-121

3. Blomberg AM, Hylander I, TornkvistL (2008) District nurses' involvement in pain care: a theoretical model. JClin Nurs 17:2022-2031

4. Bohnsack R (2009) Gruppendiskussion. In: Flick U, von Kardoff E, Steinke I (Hrsg) Qualitative Forschung. Ein Handbuch. Rohwohlt, Reinbeck bei Hamburg, S369-383

5. Breivik H, Cherny N, Collett B et al (2009) Cancerrelated pain: a pan-European survey of prevalence, treatment, and patient attitudes. Ann Oncol 20:1420-1433

6. Brockhoff R, Lempe M (2013) Die aktuelle Situation der ambulanten Pflegedienste. https://www.caritas.de/neue-caritas/heftarchiv/ jahrgang2013/artikel/die-aktuelle-situation-derambulanten-pf.Zugegriffen: 19. Dez. 2017

7. Bundesministerium der Justiz (2009) SGBXI-§112 Qualitätsverantwortung. http://www.gesetzeim-internet.de/sgb_11/_112.html.Zugegriffen: 19. Dez. 2017

8. Chang M, Chang Y, Chiou J et al (2002) Overcoming patient-related barriers to cancer pain management for home care patients: a pilot study. Cancer Nurs 25:470-476

9. De Wit R, Van Dam F (2001) From hospital to home care: a randomized controlled trial of a pain education programme for cancer patients with chronic pain. J Adv Nurs 36:742-754

10. Deutsches Netzwerk für Qualitätsentwicklung in der Pflege (2011) Expertenstandard in der Pflege beiakuten Schmerzen. DNQP, Osnabrück

11. Deutsches Netzwerk für Qualitätsentwicklung in der Pflege (2005) Expertenstandard Schmerzmanagement in der Pflege bei akuten oder tumorbedingten chronischen Schmerzen. DNQP, Osnabrück

12. Deutsches Netzwerk für Qualitätsentwicklung in der Pflege (2014) Expertenstandard Schmerzmanagement in der Pflege bei chronischen Schmerzen. DNQP, Osnabrück

13. Ferrell BR, Taylor EJ, Grant M et al (1993) Pain management at home: struggle, comfort, and mission. Cancer Nurs 16:169-178

14. Gerken L, Windisch A, Thalhammer R et al (2017) Pflegerisches Schmerzassessment aus Patientenperspektive. Schmerz 31:123-130

15. Gibbins J, Bhatia R, Forbes K et al (2014) What do patients with advanced incurable cancer want from the management of their pain? A qualitative study. Palliat Med 28:71-78

16. Glajchen M, Bookbinder M (2001) Knowledge and perceived competence of home care nurses in pain management: a national survey. J Pain Symptom Manage 21:307-316

17. Hakonsen GD, Strelec P, Campbell D et al (2009) Adherence to medication guideline criteria in cancer pain management. J Pain Symptom Manage 37:1006-1018

18. Hibbeler B (2011) Ärzte und Pflegekräfte: Ein chronischer Konflikt. Dtsch Arztebl 41:A2138-A2144

19. Krutter S, Nestler N, Elsner F et al (2016) Die Schmerzsituation von Patienten mit Tumorschmerzen in Hospizen - Ergebnisse einer qualitativen Studie in Münsterschen Hospizen. ZPalliativmed 17:243-252

20. Leiske M, Lahmann NA, Lindena G et al (2015) Schmerz bei Patienten in der ambulanten Pflege. Eine bundesweite Querschnittsstudie mit Pfadmodell. Schmerz 29:431-439

21. Mayring $P$ (2003) Einführung in die Qualitative Sozialforschung. Beltz, Weinheim, Basel

22. Mayring $P$ (2010) Qualitative Inhaltsanalyse. Grundlagen und Techniken. Beltz, Weinheim, Basel

23. Medizinischer Dienst des Spitzenverbandes Bund der Krankenkassen e.V. (2014) PflegeQualitätsbericht des MDS nach § 118Abs.4 SGBXI. Qualität in der ambulanten und stationären Pflege. MDS, Essen

24. Mehta A, Chan LS (2008) Understanding of the concept of "Total pain": a prerequisite for pain control.J Hosp Palliat Nurs 10:26-34

25. Oliver JW, Kravitz RL, Kaplan SH et al (2001) Individualized patient education and coaching to improve pain control among cancer outpatients. JClin Oncol 19:2206-2212

26. Osterbrink J, Ewers A, Nestler $\mathrm{N}$ et al (2010) Versorgungsforschungsprojekt „Aktionsbündnis Schmerzfreie Stadt Münster". Zielsetzung und Methodik. Schmerz 24:613-620

27. Perner A, Götze H, Stuhr C et al (2010) Ambulante Palliativversorgung von Tumorpatienten im Raum Leipzig. Schmerz 24:38-45

28. Randall-David E, Wright J, Porterfield DS et al (2003) Barriers to cancer pain management: homehealth and hospice nurses and patients. Support Care Cancer 11:660-665

29. Sachverständigenrat zur Begutachtung der Entwicklungen im Gesundheitswesen (SVR) (2014) 8.3 Empfehlung für regional differenzierte pflegerische Versorgungskonzepte. Neue Formen der Koopertation und Arbeitsteilung 232. http://www svr-gesundheit.de/index.php?id=521. Zugegriffen: 19.Dez. 2017

30. Schwabe S, Ates G, Hasselaar J et al (2017) „Etwas mehr Vertrauen, dass ich es schaffe .... ZPalliativmed 18:90-96

31. Statistisches Bundesamt (2017) Pflegestatistik 2015. Pflege im Rahmen der Pflegeversicherung. Ländervergleich - Ambulante Pflegedienste. DeStatis, Bonn

32. Vallerand AH, Anthony M, Saunders MM (2005) Home care nurses' perceptions of control over cancer pain. Home Healthc Nurse 23:647-654

33. Vallerand AH, Hasenau SM, Templin T (2004) Barriers to pain management by home care nurses. Home Healthc Nurse 22:831-840

34. Vallerand AH, Riley-Doucet $C$, Hasenau SM et al (2004) Improving cancer pain management by homecare nurses. Oncol Nurs Forum 31:809-816
Hier steht eine Anzeige. Springer 